\title{
Nonlinear Dynamics of Aeolian Sand Ripples
}

\author{
Leonid Prigozhin \\ Center for Energy and Environmental Physics, Blaustein Institute for Desert Research, \\ Ben Gurion University of the Negev, Sede Boqer Campus, 84990 Israel
}

(September 13, 2018)

\begin{abstract}
We study the initial instability of flat sand surface and further nonlinear dynamics of wind ripples. The proposed continuous model of ripple formation allowed us to simulate the development of a typical asymmetric ripple shape and the evolution of sand ripple pattern. We suggest that this evolution occurs via ripple merger preceded by several soliton-like interaction of ripples.
\end{abstract}

\section{INTRODUCTION}

Aeolian (i.e. wind driven) sand ripples form nice regular patterns on coastal beaches and desert floors and indicate an instability of flat sand surface under the wind-induced transport and rearrangement of loosely packed sand grains. Following fundamental work by Bagnold [1] more than half a century ago, formation of sand ripples has been studied by many researchers (see review [2] and the references therein). Significant progress in understanding the nature of this phenomenon has been achieved. Nonetheless, major questions remain open; these involve the most interesting part of ripple formation, the nonlinear interactions that follow the initial instability. Previous research on ripples has generally relied on highly simplified continuum models or on stochastic or molecular dynamics simulation. By means of a new deterministic continuous model that seems to better describe the essential physics, we here investigate salient nonlinear properties of ripple formation.

As is well known, Aeolian ripples are oriented perpendicularly to the wind direction. Mature ripples are asymmetrical in cross section: their stoss (upwind) slopes are typically much less steep than the shorter lee (downwind) slopes [2 4 . The steepness of the lee slopes cannot exceed and usually does not reach the sand angle of repose. The ripples have convex stoss slopes, concave lee slopes, and flattened crests which usually end with a brink. Since smaller ripples of the same shape have smaller volume to surface ratios, they are translated faster by the wind and can overtake the larger ripples. A possible merger results in gradual elimination of small ripples and in growth of ripple wavelength.

To analyze the mechanics of sand transport, which occurs whenever the wind is sufficiently strong, it is convenient to distinguish two types of sand grain movement: saltation and reptation (or creep) [1,2]. Saltating grains move by long trajectories that end in high-energy impacts with the surface. These impacts take place at almost uniform shallow angles of descent varying from 9 to $15^{\circ}$ to the horizontal depending on the wind strength and grain size [5]. After an impact, a saltating grain usually rebounds sufficiently high to be accelerated by the wind again and continues its saltation. These grains gain energy from the wind and transfer part of it to the sand bed on impact. Each impact may cause ejection of one or several low energy (reptating) particles from the bed surface. Reptating particles make a short hop, usually jumping or rolling for several millimeters or less, and stop. The exchange flux between saltating and reptating grain populations is supposed to be small [6,7].

According to the hypothesis put forward by Bagnold [1], the ripple wavelength is equal to the mean length of saltation jump. However, this claim has been challenged by numerous researchers (see, e.g., [2, []) and it is now commonly accepted that the essential physics lies in the variation of reptation flux. The role of saltation, whose trajectories are many times longer than the ripple wavelength, is indirect. The oblique, almost unidirectional bombardment by saltating particles supplies the energy necessary for reptation. Wind strength determines the intensity of saltation; the probability of direct entrainment of particles into reptation by wind is small [1].

A model, based on these views, was proposed by Anderson [6]. Linear stability analysis of this model showed that the initial ripple wavelength is determined by, and is several times larger than, the mean length of reptation. Unfortunately, the model yields unrealistic results at the nonlinear stage of ripple growth, which begins very early. It has been suggested [2] that the model can be improved by allowing the reptating grains to continue rolling upon the bed surface after landing, and not to stop immediately as was assumed originally. Although no such continuous model has been developed, employing a similar approach in molecular dynamics computer simulations of sand ripples [7] was quite successful.

Interesting results on modeling different aspects of sand ripples dynamics have been obtained by means of cellular automata models or molecular dynamics simulations [7-10]. It was even claimed [10] that no continuum mechanics or deterministic model can capture the main feature of ripple self-organization: the increase of scale in time due 
to merging of ripples. Below, we show that this general conclusion, based on a schematic discrete model of ripple merging, is wrong. The continuous deterministic model proposed herein provides a better description of the physical process than the simplified stochastic model [10]. Our model reproduces the asymmetrical ripple shape and is able to simulate not only merging of ripples but also a more complicated, soliton-like mode of ripple interaction which can be equally significant for ripple self-organization. A somewhat similar behavior has been observed in molecular dynamics simulation of sand ripples [7].

Let us mention here also the analytical model of sand ripples [11]. In this model, the upwind slope of a ripple is given by a smooth solution of the diffusion equation with a negative diffusion coefficient. Such a solution is, however, unstable. Furthermore, in the absence of wind the ripple shape in [11] is described by a well-posed diffusion equation. This is also unphysical: according to this model ripples diffuse and disappear, since surface particles continue to roll down the slopes. Real sand ripples are, of course, metastable.

To derive a model allowing for metastability, it is necessary to take into account that the surface flux of granular material is not determined solely by the local surface slope. Models involving an additional variable, surface flux or the density of rolling particles, have recently been derived to simulate quasistationary evolution of a growing pile shape 12] or to model the dynamics of pile surface in more detail and on a shorter spatio-temporal scale ( 13 , , see also [14]). In our model of Aeolian ripples we use a similar approach to describe the flow of rolling particles.

\section{MATHEMATICAL MODEL}

In accordance with the physical picture of sand transport described above, we assume that there exists a uniform flux of saltating grains which reach the bed surface $y=h(\mathbf{x}, t)$ at a low angle $\gamma$ to horizontal. The impacts cause erosion of this surface. The erosion rate $f$ is proportional to the impact intensity, which depends on the surface orientation with respect to the direction of saltation. Let the saltating particles strike an inclined surface at an angle $\theta$. Then $f$ is proportional to $\sin \theta$ and we can write

$$
f=f_{0} \frac{\sin \theta}{\sin \gamma}
$$

where $f_{0}$ is the rate of erosion of a horizontal surface (determined by the intensity of saltation). However, if the surface has sufficiently steep slopes, some parts of it may be in shadow and unreachable by saltating grains. In this case we set $f=0$; shadowing introduces non-locality into this problem.

A reptating particle, ejected by an impact at a point $\mathbf{x}$, makes a jump and lands on the bed surface at a point $\mathbf{y}$ with probability density $p$ given by the "splash function", $p=p_{\alpha}(\mathbf{x}, \mathbf{y})$, first introduced in [15]. This function, which will be specified below, depends, in particular, on the surface slope at the point of impact. In our model, the splash function also accounts for all the anisotropy induced by a chosen wind (saltation) direction.

Upon landing, reptating particles do not stop immediately but may roll away, although usually not far from the landing point. Let $R(\mathbf{x}, t)$ be the effective surface density of rolling particles ( $R d \mathbf{x}$ is the volume which particles, presently rolling over the part of the free surface above the area $d \mathbf{x}$, would occupy in the sand bed). When they stop, the rolling particles are incorporated into the motionless bed. Following [13], we denote by $\Gamma[h, R]$ the rate of rolling-to-steady state transition and write the mass conservation equations for the sand bed and for the population of rolling particles:

$$
\begin{aligned}
\partial_{t} h & =\Gamma[h, R]-f, \\
\partial_{t} R+\nabla \cdot \mathbf{J} & =Q-\Gamma[h, R] .
\end{aligned}
$$

Here $\mathbf{J}$ is the horizontal projection of the flux of rolling particles, and the source term

$$
Q(\mathbf{x}, t)=\int f(\mathbf{y}, t) p_{\alpha}(\mathbf{y}, \mathbf{x}) d \mathbf{y}
$$

gives the intensity of "rain" of falling reptating particles.

We assume that reptating particles lose most of their momentum in collision with the rough bed surface. Neglecting inertia, we postulate that upon landing the particles roll in direction of the steepest descent and that the steeper the slope the faster they roll. The simplest form [16] of the flux $\mathbf{J}$ is, therefore,

$$
\mathbf{J}=-\mu_{0} R \nabla h,
$$

where $\mu_{0}$ is a constant "mobility" of particles. 
The rate of rolling-to-steady state transition, $\Gamma$, depends on stability of a particle on inclined sand bed surface and on the amount of rolling particles. Rolling particles never form a thick layer on the surface during the ripple growth: there is only a small amount of them at each time moment. It can be assumed that, for a fixed free surface incline, the rate of rolling-to-steady state transition is proportional to the amount of rolling particles on the surface, R. Since the exchange rate cannot depend on the free surface slope orientation, we further assume $\Gamma$ is a (smooth) function of $|\nabla h|^{2}$. The steeper the free surface is, the easier do particles roll down and, correspondingly, the lower is the rate of rolling-to-steady state transition. For slopes steeper than the sand angle of repose, $\alpha_{r} \approx 30^{\circ}$, rolling sand grains do not stop at all. Taking these arguments into account we assume, as a simple but physically reasonable approximation, that $\Gamma$ is also proportional to $\tan ^{2} \alpha_{r}-|\nabla h|^{2}$ and obtain

$$
\Gamma=\kappa_{0} R\left(1-\frac{|\nabla h|^{2}}{\tan ^{2} \alpha_{r}}\right),
$$

where $\kappa_{0}$ characterizes particle stability upon a horizontal surface.

We now rescale the variables,

$$
t^{\prime}=f_{0} t, \quad R^{\prime}=\frac{\kappa_{0}}{f_{0}} R, \quad f^{\prime}=\frac{1}{f_{0}} f, \quad \mathbf{J}^{\prime}=\frac{1}{f_{0}} \mathbf{J}, \quad \Gamma^{\prime}=\frac{1}{f_{0}} \Gamma,
$$

and obtain

$$
\mathbf{J}=-\nu R \nabla h, \quad \Gamma=R\left(1-\frac{|\nabla h|^{2}}{\tan ^{2} \alpha_{r}}\right)
$$

and $f=\sin \theta / \sin \gamma$, or $f=0$ in a shadow. Here $\theta=\theta(\mathbf{x}, t)$ is the angle at which the saltating particles strike the bed, and $\nu=\mu_{0} / \kappa_{0}$ is a constitutive dimensionless parameter characterizing the competition between mobility and stability of dislodged grains on the sand surface. The rescaling leaves the equation (11) invariant while the equation (2) takes the form

$$
\frac{f_{0}}{\kappa_{0}} \partial_{t} R+\nabla \cdot \mathbf{J}=Q-\Gamma[h, R] .
$$

We suppose that the rate of sand surface erosion caused by saltation, $f_{0}$, is usually much smaller than $\kappa_{0}$, the coefficient determining the rate at which rolling particles come to a stop on the horizontal surface and are absorbed by the motionless bulk (that is why the layer of rolling particles is so thin). Neglecting the small term in equation (5) we arrive at a quasistationary mass balance equation for rolling particles,

$$
\nabla \cdot \mathbf{J}=Q-\Gamma[h, R] .
$$

\section{SPLASH FUNCTION}

To complete the model (11), (3), (1), and (6) we need to specify the splash function. Although not much is known about this function, previous studies (see, e.g., [6 8 ) suggest that the system is not very sensitive to the details of splash function behavior and that an approximation, sufficient at least for qualitative simulation, may be obtained by combining the existing experimental data and simple physical arguments. We limit our consideration to the one-dimensional (1D) case.

Collision of quartz grains with a sand bed has been studied experimentally by Willetts and Rice [5]. It was found that ejection of reptating grains from the bed depends only slightly on the incident angle of attack, which varied in these experiments in accordance with the systematic changes of the bed inclination. (The angle of descent of saltating particles to the horizontal was constant). For various incident angles, ejection occurred at approximately the same mean angle to the bed surface, $m_{\phi} \approx 50^{\circ}$, with standard deviation $\sigma_{\phi} \approx 40^{\circ}$, and the same mean velocity of ejecta.

We use these results to crudely reconstruct the dependence of the splash function on the bed surface inclination in 1D case. First, we define (somewhat arbitrarily, see [17]) the density of ejection angle distribution, $p=p(\phi)$, providing for the mean and standard deviation values as found in [5] (see Fig. 1a). For simplicity, we further assume that particles are ejected from the bed with the same initial velocity $v_{0}$ at different angles $\phi$, that they then follow simple ballistic trajectories $x=x_{0}+v_{0} \cos (\alpha+\phi) t, y=h\left(x_{0}\right)+v_{0} \sin (\alpha+\phi) t-\frac{1}{2} g t^{2}$, and hit the surface at a horizontal distance $s$ from the ejection point. Here $\alpha=\tan ^{-1}\left(\partial_{x} h\right)$ is the surface angle at the ejection point $x_{0}, g$-acceleration 
of gravity. Assuming the surface curvature is small, $\left|\partial_{x x}^{2} h\right| \ll g / v_{0}^{2}$, we approximate $h\left(x_{0}+s\right)$ by $h\left(x_{0}\right)+s \partial_{x} h\left(x_{0}\right)$ and find the jump length

$$
s=\frac{v_{0}^{2}}{g}\left(\sin 2\{\alpha+\phi\}-2 \cos ^{2}\{\alpha+\phi\} \tan \alpha\right)
$$

Making use of the probability density $p(\phi)$, we can now calculate numerically the mean and the standard deviation of reptation jump length, $m_{r}(\alpha)$ and $\sigma_{r}(\alpha)$, for any bed surface inclination $\alpha$, up to the value of a proportionality coefficient $v_{0}^{2} / g$. This factor is eliminated from the final dimensionless formulation of the model by choosing the unit of length equal to the mean reptation length at the horizontal bed surface. Thus $m_{r}(0)=1$ by definition. The functions $m_{r}(\alpha)$ and $\sigma_{r}(\alpha)$ are shown in Fig. 1b. Finally, we approximate the splash function $p_{\alpha}$ by the density of a corresponding normal distribution,

$$
p_{\alpha}\left(x_{0}, y\right)=\frac{1}{\sigma \sqrt{2 \pi}} \exp \left(-\frac{1}{2}\left[\frac{y-x_{0}-m}{\sigma}\right]^{2}\right),
$$

where $\sigma=\sigma_{r}(\alpha), m=m_{r}(\alpha)$, and $\alpha=\tan ^{-1} \partial_{x} h\left(x_{0}, t\right)$.

\section{LINEAR STABILITY ANALYSIS}

To analyze the initial instability of a horizontal sand bed, we assume that $h$ and its derivatives are small and linearize the model. Up to the second order terms, $\Gamma[h, R]=R$ and $f=1+k_{\gamma} \partial_{x} h$, where $k_{\gamma}=\cot \gamma$, so Eq. (1) yields $R=1+\partial_{t} h+k_{\gamma} \partial_{x} h$.

For small surface slopes, the standard deviation of reptation length does not change much (see Fig. 1b) and we set $\sigma_{r} \equiv \sigma_{r}(0)=1.25$. Knowing the dependence $m_{r}(\alpha)$ (Fig. 1b), it is easy to find numerically that for small slopes $m_{r} \approx 1-k_{m} \partial_{x} h$, where $k_{m}=2.01$.

Let $p_{0}(x)$ be the density of the normal distribution $N\left(1, \sigma_{r}^{2}(0)\right)$. It is not difficult to show that $p_{\alpha}(x, y)=p_{0}(y-$ $x)+k_{m} p_{0}^{\prime}(y-x) \partial_{x} h(x)$ plus the higher order terms ("'" means derivative). The linearized Eq. (3) takes the form $Q=1+k_{\gamma} p_{0} * \partial_{x} h+k_{m} p_{0}^{\prime} * \partial_{x} h$, where "*" is the operator of convolution.

Substituting the linear approximations for $\Gamma, R$, and $Q$ into Eq. (6) we obtain, up to the second order terms,

$$
\partial_{t} h=\nu \partial_{x x}^{2} h+k_{\gamma}\left(p_{0} * \partial_{x} h-\partial_{x} h\right)+k_{m} p_{0}^{\prime} * \partial_{x} h
$$

We can now apply the Fourier transform and substitute $h=e^{\lambda t+i \omega x}$ to find the dispersion relation

$$
\lambda(\omega)=-\nu \omega^{2}+k_{\gamma} i w\left(\widetilde{p}_{0}-1\right)-k_{m} \omega^{2} \widetilde{p}_{0},
$$

where $\widetilde{p}_{0}=\exp \left(-\left[\omega \sigma_{r}(0)\right]^{2} / 2-i w\right)$ is the Fourier transform of $p_{0}$. Note that with $\nu=k_{m}=0$ one gets the dispersion relation for Anderson's model [6]. The initial ripple wavelength can be calculated as $l_{0}=2 \pi / \omega_{0}$, where $\omega_{0}$ is the wave number at which the expression

$$
\operatorname{Re} \lambda(\omega)=-\nu \omega^{2}+\omega\left(k_{\gamma} \sin \omega-k_{m} \omega \cos \omega\right) e^{-\left(\omega \sigma_{r}(0)\right)^{2} / 2}
$$

attains a positive maximum. This maximum exists and the flat surface is unstable if

$$
k_{\gamma}>k_{m}+\nu .
$$

To explain this result we note that ripples grow because of the geometrical effect of greater impact and ejection flux on upwind-oriented slopes than on downwind-oriented slopes [8]. This non-uniformity increases if the saltation angle of attack becomes smaller ( $k_{\gamma}$ increases). On the other hand, the greater $\nu$ and $k_{m}$ are, the more significant are, respectively, smoothing effects due to rolling of dislodged particles down the surface slopes and scattering of ejected particle trajectories by an uneven sand surface. Condition (7) indicates when the instability prevails.

As it was mentioned above, the saltating particles usually hit the sand surface at almost uniform angle to the horizontal, varying from 9 to $15^{\circ}$. In the examples below we use the mean reported value of this angle, $\gamma=12^{\circ}$. Using the stability condition (耳), it is easy to calculate that the flat surface is unstable if $\nu<2.69$. 


\section{NONLINEAR DYNAMICS}

To study the ripple evolution further we solved the nonlinear system (11), (3), (4), (6) numerically, assuming periodic boundary conditions and using an implicit finite-difference approximation. The initial evolution of a slightly disturbed flat surface obeys the linear theory: after a short initial stage the fastest growing mode, having the wavelength predicted by the linear stability analysis, dominates.

The growing ripples remain almost symmetric until, shortly before the appearance of first shadow regions, the downwind slopes become steeper. This asymmetry develops quickly as the ripples continue to grow. Further evolution is accompanied by the increase of the ripple wavelength (see Fig. 2). It can be seen that the downwind translation of ripples gradually slows down as their size increases. Although our model is much simplified in many respects, the calculated mature ripple shape is similar to that of the real sand ripples [3]: for $\nu=2$ we obtained convex stoss slopes inclined at about $11-13^{\circ}$, flattened crests, and slightly concave lee slopes with the mean maximal inclination $26-27^{\circ}$; the ripple index (length to height ratio) was about 14 . For $\nu=1$ the results are qualitatively similar, although the mean maximal inclinations are $15^{\circ}$ and $28-29^{\circ}$ for the stoss and lee slopes, respectively, and the ripple index is smaller.

The most interesting part of ripple dynamics is the mechanism of ripple merging and self-organization. The simulations show that simple merger takes place only if the overtaking ripple is much smaller than the bigger "overtaken" ripple, which moves more slowly. Otherwise another, more complicated scenario is usually realized. As a smaller ripple reaches the larger, the trough between them becomes shallow and a "two-headed" long ripple appears. Then the "downwind head", which originally formed the larger ripple crest, starts to move forward as a separate small ripple and runs ahead. Two new ripples emerge from this recombination. The ripple that is left behind is larger than the larger of the two ripples before merger. The ripple that runs away is smaller than the smaller one before this event (Fig. 3). Sometimes the trough between the two merging ripples disappears before the generated long ripple becomes unstable. However, soon there appears a new trough near the end of this ripple crest and a small running away ripple develops. Since it is smaller, this ripple proceeds even faster and soon meets another large ripple on its way. Complete merger is now more probable, since the overtaking ripple became smaller. However, another recombination may yet occur before the material redistribution between ripples is completed. Obviously, such a mechanism of ripple interaction produces a regular ripple array structure more efficiently than a simple merger of ripples.

In our opinion, this scheme of ripple interaction gives also a likely explanation to the appearance of small secondary ripples in wind tunnel experiment [18]. Indeed, the appearance of such ripples due to the backward eddy flow behind a ripple, as is suggested in [18], seems hardly possible. Sand ripples are so shallow that most probably there is no backward flows in their shadows. Even if small backward eddies existed, they could never cause saltation of sand grains against the main wind direction, and thus could not produce any sand ripples. As follows from our simulation, no backward flows are necessary: small ripples appear as a means of redistribution of mass during the ripple array reconstruction leading to the wavelength growth.

\section{ACKNOWLEDGMENTS}

The author appreciates discussions with Yu. Shtemler and I. Rubinstein. This work was supported by the Blaustein International Center for Desert Studies.

[1] R. A. Bagnold, The physics of Blown Sand and Desert Dunes (Methuen and Co., London, 1941).

[2] R. S. Anderson, Earth-Sci. Rev. 29, 77 (1990).

[3] R. P. Sharp, J. Geol. 71, 617 (1963).

[4] B. T. Werner, P. K. Haff, R. P. Livi, and R. S. Anderson, Geology 14, 743 (1986).

[5] B. B. Willetts and M. A. Rice, Earth Surf. Processes \& Landforms 14, 719 (1989).

[6] R. S. Anderson, Sedimentology 34, 943 (1987).

[7] W. Landry and B. T. Werner, Physica D 77, 238 (1994).

[8] S. B. Forrest and P. K. Haff, Science 255, 1240 (1992).

[9] R. S. Anderson and K. L. Bunas, Nature 365, 740 (1993); H. Nishimori and N. Ouchi, Phys. Rev. Lett. 71, 197 (1993); N. B. Ouchi and H. Nishimori, Phys. Rev. E 525877 (1995).

[10] B. T. Werner and D. T. Gillespie, Phys. Rev. Lett. 71, 3230 (1993). 
[11] R. B. Hoyle and A. W. Woods, Phys. Rev. E 56, 6861 (1997).

[12] L. Prigozhin, Zh. Vychisl. Mat. Mat. Fiz. 26, 1072 (1986); Phys. Rev. E 49, 1161 (1994); Chem. Eng. Sci. 48, 3647 (1993).

[13] J. P. Bouchaud, M. E. Cates, J. Ravi Prakash, and S. F. Edwards, J. Phys. I France 4, 1383 (1994); Phys. Rev. Lett. 74, 1982 (1995).

[14] A. Mehta and G. Barker, Phys. Rev. Lett. 67, 394 (1991); A. Mehta, J. M. Luck, and R. J. Needs, Phys. Rev. E 53, 92 (1996).

[15] J. E. Ungar and P. K. Haff, Sedimentology 34, 289 (1987).

[16] The diffusion component of surface flux, $-D \nabla R$, is omitted. We found that if such a term is made significant, the model yields a somewhat distorted ripple shape.

[17] This function has been identified by the known mean and variance values from a two-parametric family of probability densities, $p(\phi)=C \exp \left(-\frac{1}{2}\left\{\frac{\phi-a}{b}\right\}^{2}\right)$, where $C$ is the normalizing coefficient and $0 \leq \phi \leq 180^{\circ}$. The identified values of parameters are: $a=-40^{\circ}, b=80^{\circ}$.

[18] M. Seppälä and K. Lindé, Geografiska Annaler 60A, 29 (1978).

FIG. 1. $a$-density of ejection angle distribution, $p(\phi) ; b$ - the mean (solid line) and standard deviation (dashed line) of reptation jump length as functions of surface inclination.

FIG. 2. Flat surface instability and formation of sand ripples. Wind direction is from left to right; the unit of length is the mean length of the reptation jump; $\nu=2$.

FIG. 3. A typical ripple interaction; see the region bounded by the thin line. To show the details the ripples are stretched in the vertical direction. 

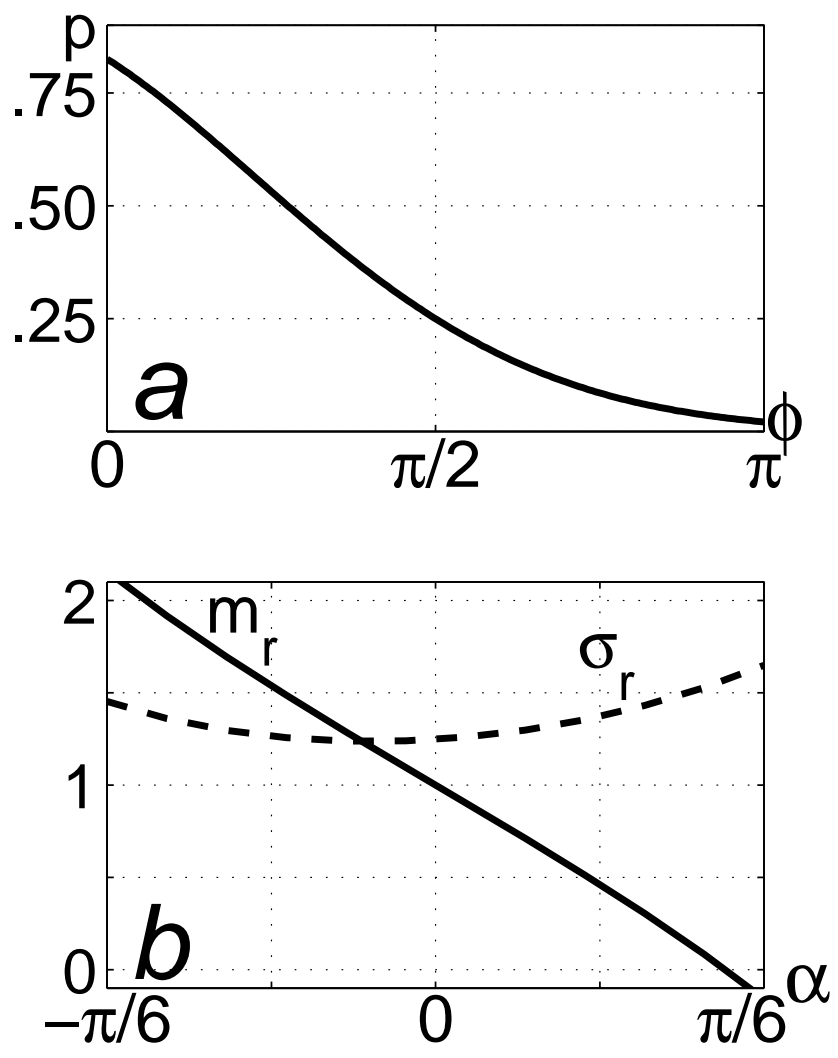


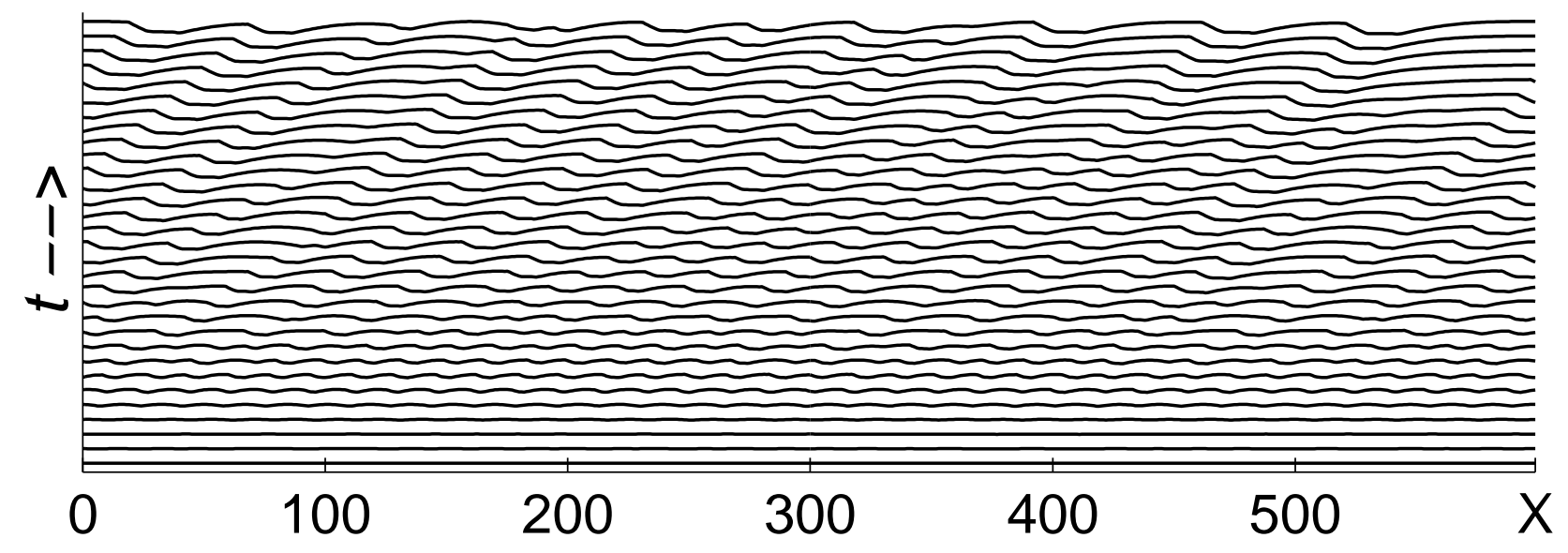




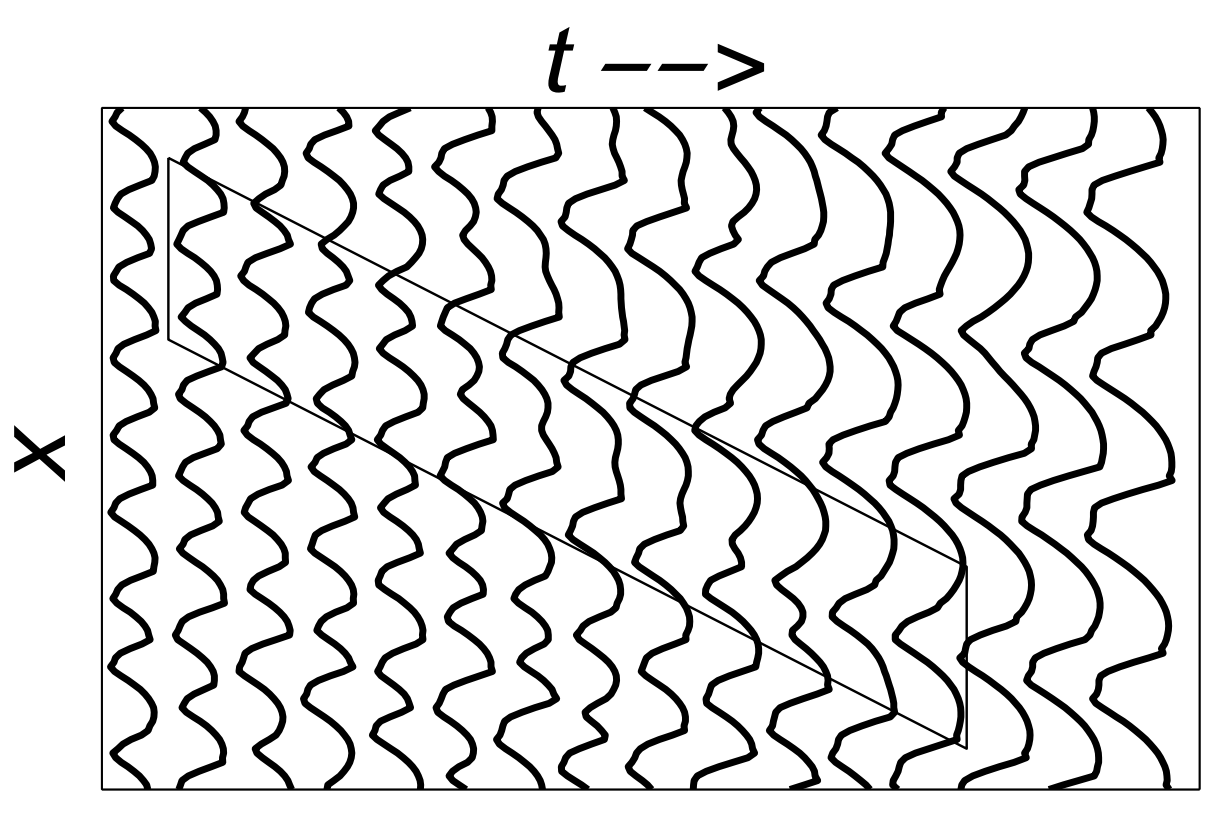

